\title{
Maximum-Weight Markings in Marked Graphs: Algorithms and Interpretations Based on the Simplex Method
}

\author{
K. THULASIRAMAN, SENIOR MEMBER, IEEE, AND MARC A. COMEAU, MEMBER, IEEE
}

\begin{abstract}
The problem of determining a maximum-weight marking in a marked graph is mathematically dual to the transshipment problem of operations research. The special structure of the transshipment problem facilitates efficient implementation of the simplex method of linear programming, for solving such problems. In this paper, we first show that the maximum-weight marking problem possesses as much structure as its dual, and then present an implementation of simplex for this problem in terms of marked graph concepts and operations. The pivoting operation in the simplex method is shown to correspond to the subgraph firing operation in marked graphs. A diakoptic reachability theorem is also proved. The formulations presented in this paper cover both live- and nonlive-marked graphs with or without capacity constraints.
\end{abstract}

\section{INTRODUCTION}

Petri net is a general algebraic structure originally developed by Carl Adam Petri as a model for information flow in systems exhibiting asynchronism and parallelism [1]. The generality of the Petri net makes modeling of complex networks possible. However, the feasibility of analysis becomes questionable and in many cases the problems are NP-Complete. Marked graphs are a special class of Petri nets, which are more amenable to analysis; yet they retain enough generality to model systems of parallel processing, queueing networks, resources allocation schemes and many other related problems. In the theory of marked graphs, a main concern is the study of possible token distributions at markings which are reachable from an initial marking. Various issues have been studied in this context [2]-[6]. In this paper, we formulate and study a maximization problem defined on a marked graph.

In the following section, we review some of the results on marked graphs on which is based the remainder of this paper. In Section III, we define the maximization problem, namely, the maximum-weight marking problem and provide an algorithmic solution to this problem. Our algorithm is based on the simplex method of linear programming [7]. As we develop our main algorithm, we offer interpretations of operations which one encounters in the theory of linear programming, in terms of marked graph concepts. In Section IV, we extend the results of Section

Manuscript received March 21, 1986; revised January 7, 1987. This work was reported part of the Ph.D. dissertation of the second author, M. A. Comeau, Concordia University, Montreal, Canada, June 1986.

K. Thulasiraman is with the Department of Electrical and Computer

Engineering, Concordia University, Montreal, P.Q., Canada H3G $1 \mathrm{M} 8$.

M. A. Comeau is with the Centre de Recherche Informatique de Montreal, Inc., Montreal, P.Q., Canada, and also with the Department of Electrical Engineering, Concordia University, Montreal, P.Q., Canada. IEEE Log Number 8717306.
III to the capacitated case. In this section, we also provide an alternative formulation of the problem, suitable for studying the nonlive case which is presented in Sections $\mathrm{V}$ and VI.

\section{Prelude}

A marked graph [1] is defined as a directed graph $G=(V, E)$ with vertex set $V$, edge set $E$, a nonnegativeinteger column vector $M$ associated with $E$ called a marking, state or token distribution of $G$, and a state-transition function $\delta_{i}(M)$ mapping $M$ into a new marking $M^{\prime}$ resulting from firing vertex $i \in V$. The transition function subtracts one token from $M$ on each edge incident into $i$ and adds one token to $M$ on each edge incident out of $i$, to obtain $M^{\prime}$. Since $M^{\prime}$ must be nonnegative, $\delta_{i}(M)$ can only be applied if $M$ has a positive token count on each edge incident into vertex $i$. Vertex $i$ is said to be enabled if $\delta_{i}(M)$ can be applied.

A marking $M^{\prime}$ is reachable from a marking $M$ if a sequence of legal transitions will transform $M$ into $M^{\prime}$. The reachability set $R(M)$ of a marking $M$ is defined as the set of all markings reachable from $M$. Since the null sequence is trivially legal, $M \in R(M)$.

A marked graph is live under a marking $M$ if each vertex $i \in G$ can be enabled through some legal firing sequence starting from $M$. Liveness is characterized in the following theorem [2].

Theorem 1: A marked graph $G$ is live under a marking $M$ if and only if $G$ contains no token-free directed circuits under $M$.

Let $G$ be a marked graph with an initial marking $M_{0}$ and let $M \in R\left(M_{0}\right)$. Then, the differential marking $\Delta_{M} \triangleq$ $M-M_{0}$ satisfies Kirchoff's voltage law in $G$ [3]. If $B_{f}$ is a fundamental-circuit matrix of $G$ then $B_{f} \Delta_{M}=0$. This simple and elegant result, as profound as Tellegen's theorem, is the basis of all the results presented in this paper. A token-free directed circuit of $G$ under $M_{0}$ is called a dead-circuit of $G$. In these terms, reachability is characterized in the following theorem [3].

Theorem 2 (Reachability Theorem): A marking $M$ of a marked graph $G$ is reachable from an initial marking $M_{0}$ of $G$ if and only if $B_{f} \Delta_{M}=0$ and $\sigma_{v}=0$ for each vertex $v$ belonging to a dead-circuit of $G$, in the minimum nonnegative solution of $A^{t} \Sigma=\Delta_{M}$ where

$$
\Sigma=\left[\sigma_{1}, \sigma_{2} \cdots \sigma_{n}\right]^{t}
$$

and $A$ is the incidence matrix of $G$. 
The $\sigma_{i}$ 's referred to in the above theorem are called the firing numbers or firing counts of the corresponding vertices.

A marked graph $G$ is bounded under a marking $M_{0}$ if the token count on each edge, $e \in G$, is finite in every marking in $R\left(M_{0}\right)$. Boundedness is guaranteed if and only if each edge of $G$ belongs to a directed circuit with a finite token count [2]. The marked graph is called $k$-bounded if $M^{\prime}(e) \leqslant k, \forall e \in G, \forall M^{\prime} \in R(M)$. A 1-bounded marked graph is called safe.

\section{The Maximum-Weight Marking Problem}

Given a marked graph $G=(V, E)$ with an initial marking $M_{0}$, we consider the problem of obtaining an $M \in$ $R\left(M_{0}\right)$ which is maximum or minimum in some sense. An obvious objective is simply $\Sigma_{e} M(e)$, the token count of $G$ under $M$. In fact, a solution to this problem for live, strongly connected marked graphs can be found in [2], where the authors employ a circulation-flow approach for solving its dual. A linear-programming formulation of a more general problem, namely, the maximum-weight marking problem and its dual was presented by Murata in [8]. In this paper, we focus on this generalized problem. Thus we introduce a per token weight $W(e)$ associated with each edge $e \in E$ which represents the weight or cost of one token residing there and consider the maximumweight marking problem

$$
\underset{M \in R\left(M_{0}\right)}{\operatorname{maximize}} W M
$$

where $W$ is the row vector of edge weights. We would like to point out that the problem of determining a maximum weight marking in a marked graph is equivalent to the problem of determining a maximum marking in a computation graph when the input quantum, the output quantum and the threshold of each edge of the computation graph are all equal [9].

Our discussion begins with a linear-programming formulation of the maximum-weight marking problem based on the reachability theorem, namely, Theorem 2 . Let $T$ be a spanning tree of $G$ and let $\bar{T}$ be the corresponding cospanning tree of $G$. Let $B_{f}$ be the fundamental-circuit matrix of $G$ with respect to the tree $T$. Let $Z_{\bar{T}}$ be the vector $B_{f} M_{0}$. The reachability theorem provides a circuit-theoretic characterization of the reachability set of $M_{0}$ on $G$. If we relax or neglect the dead-circuit condition of the theorem by considering live-marked graphs only, then clearly, the maximum-weight marking problem is equivalent to the linear program

$$
\begin{aligned}
\operatorname{maximize} W M & \\
\text { subject to } B_{f} M & =Z_{\bar{T}} \\
M & \geqslant 0 .
\end{aligned}
$$

It is not obvious how to incorporate the dead-circut condition into this linear-program format since the deadcircuit condition involves the firing counts of the vertices of $G$ and Program 2 is stated in $M$ only. For this reason, we will focus first on the live class of problems characterized by (2). We consider in Section IV, an alternative formulation of Problem 1 in terms of the vertex firing numbers which is equivalent to (2) for the live class of problems and which captures the nature of the nonlive case presented in Section V.

\subsection{Basic Markings}

Central to the methods of linear programming, namely, the simplex method and its variants, is the concept of a basic solution to a consistent, underspecified system of independent, linear equations. For such systems, it is always possible to express a subset of the variables, called basic variables, explicitly in terms of the remaining nonbasic variables. If we specify zero values for the nonbasic variables then we obtain a basic solution - one in which only basic variables may have nonzero values. Any assignment of the variables in a linear program which satisfies all of its constraints constitutes a feasible solution. Besides the circuit equations, Program 2 has the nonnegativity constraint $M \geqslant 0$. Thus, any nonnegative solution to $B_{f} M=Z_{\vec{T}}$ is a feasible solution of (2). The feasible solutions of (2) are in one-to-one correspondence with the elements of $R\left(M_{0}\right)$.

The simplex method examines only basic feasible solutions during its search of an optimal one. Hence, we must define a basic marking. The canonical or echelon form of a fundamental-circuit circuit matrix implies that the cospanning-tree variables can be expressed in terms of the spanning-tree variables and hence, the cospanning-tree variables become the basic variables and the tree variables become the nonbasic variables. Thus we have the following definition.

A marking $M$ of $G$ is called a basic marking if there exists a token-free spanning tree of $G$ under $M$.

In order to apply the simplex method, we must obtain a basic feasible solution of (2). From the marked graph point of view, it is not even clear that there exists a basic marking $M \in R\left(M_{0}\right)$. Indeed, as can be easily seen, there may be no basic marking reachable from $M_{0}$, if $M_{0}$ is not live on $G$. However, as we will soon prove, it is always possible to obtain a basic $M \in R\left(M_{0}\right)$ for the live class of problems.

\subsection{Optimality Criterion}

In the following, we study the structure of the maximum-marking problem and derive the criterion for optimality.

The vector $Z_{\bar{T}}$ is an invariant of the equivalence class $R\left(M_{0}\right)$ with respect to $\bar{T}$, for obvious reasons. Every $M \in R\left(M_{0}\right)$ satisfies $B_{f} M=Z_{\bar{T}}$. The $k$ th component of $Z_{\bar{T}}$ is an invariant of the $k$ th fundamental circuit. It is simply the algebraic sum of the tokens in the $k$ th fundamental circuit when traversed in the direction of its defining chord. This number must be the same for every $M \in$ $R\left(M_{0}\right)$. It is easy to prove the following.

Lemma 1: $Z_{\bar{T}} \geqslant 0$ when $M_{0}$ is basic with tree $T$.

The cost or weight of a fundamental cutset is defined as the algebraic sum of its edge weights with respect to the forward orientation of the cutset. If $K=K_{+} \cup K_{-}$is a cutset with forward edge set $K_{+}$and backward edge set $K_{-}$, then we shall denote the weight of $K$ under the 
weighting $W$ of $E$ as

$$
W(K)=\sum_{e \in K_{+}} W(e)-\sum_{e \in K_{-}} W(e) .
$$

Next, let us eliminate the chord (basic) variables from the objective to obtain an equivalent objective in terms of the branch (nonbasic) variables only. To do this, we need the basic dictionary [7], which is readily available from the canonical form of the constraints. The partition is as follows:

$$
\text { maximize } J=\left[W_{\bar{T}}, W_{T}\right]\left[\begin{array}{c}
M_{\bar{T}} \\
M_{T}
\end{array}\right]=W_{\bar{T}} M_{\bar{T}}+W_{T} M_{T}
$$

subject to

$$
\left[I_{\bar{T}} B_{f T}\right]\left[\begin{array}{l}
M_{\bar{T}} \\
M_{T}
\end{array}\right]=M_{\bar{T}}+B_{f T} M_{T}=Z_{\bar{T}}, \quad M \geqslant 0
$$

where the subscript $\bar{T}$ denotes the cospanning tree, the subscript $T$ denotes the spanning tree, and $I_{\bar{T}}$ is a unit or identity matrix of dimension $|\widetilde{T}|$. The basic dictionary is simply

$$
M_{\bar{T}}=Z_{\bar{T}}-B_{f T} M_{T}
$$

Replacing the cospanning tree marking $M_{\bar{T}}$ with its dictionary, in the objective $J$, gives

$$
\begin{aligned}
J & =W_{\bar{T}}\left(Z_{\bar{T}}-B_{f T} M_{T}\right)+W_{T} M_{T} \\
& =W_{\bar{T}} Z_{\bar{T}}+\left(W_{T}-W_{\bar{T}} B_{f T}\right) M_{T} \\
& =W_{\bar{T}} Z_{\bar{T}}+\tilde{W}_{T} M_{T} .
\end{aligned}
$$

The number $W_{\bar{T}} Z_{\bar{T}}$ is the value of the original objective $J$ under the marking $M_{0}$ since the tree variables are all zero. Thus an equivalent objective for our optimization problem is

$$
\tilde{J} \triangleq \tilde{W} M
$$

where

$$
\begin{aligned}
& \tilde{W} \triangleq\left[\tilde{W}_{\bar{T}}, \tilde{W}_{T}\right] \\
& \tilde{W}_{\bar{T}} \triangleq 0 \\
& \tilde{W}_{T} \triangleq W_{T}-W_{\bar{T}} B_{f T} .
\end{aligned}
$$

We see from the expressions for $J$ and $\tilde{J}$ that we can increase their values by increasing, by an appropriate amount, the token count on any tree edgc with a corresponding positive coefficient in $\tilde{W}_{T}$. The elements of $\tilde{W}$ are the familiar relative-cost coefficients from the simplex method. It is easily seen that the objective $J$ or $\tilde{J}$ cannot be increased by increasing values of tree variables if $\tilde{W}_{T} \leqslant 0$ and hence, $M$ is optimal if and only if $\tilde{W}_{T} \leqslant 0$.

Theorem 3: The relative branch costs are the associated fundamental-cutset weights.

Proof: The relative-cost vector of the tree is

$$
\tilde{W}_{T}=W_{T}-W_{\bar{T}} B_{f T} .
$$

Transposing,

$$
\begin{aligned}
\tilde{W}_{T}^{t} & =W_{T}^{t}-B_{f T}^{t} W_{T}^{t} \\
& =I_{T} W_{T}^{t}-B_{f T}^{t} W_{T}^{t} \\
& =\left[-B_{f T}^{t} I_{T}\right]\left[\begin{array}{l}
W_{T}^{t} \\
W_{T}^{t}
\end{array}\right] \\
& =Q_{f} W^{t} .
\end{aligned}
$$

Transposing again,

$$
\tilde{W}_{\bar{T}}=W Q_{f}^{t}
$$

where $Q_{f}$ is the fundamental-cutset matrix of $G$ with respect to the tree $T$.

This result enables us to translate the algebraic criterion for optimality, $\tilde{W}_{T} \leqslant 0$, into a structural criterion for optimality, namely, all fundamental cutsets of $T$ have nonpositive weight, and exploit this structural property of an optimal solution in an efficient graph algorithm.

\subsection{Diakoptic Transitions}

We now shall extend the concept of enablement to a subgraph of $G$ and thus introduce the notion of a diakoptic transition in a marked graph.

Let $S$ and $\bar{S}=V-S$ be a partition of $V$ and let $\langle S, \bar{S}\rangle$ denote the cut $\langle S, \bar{S}\rangle_{+} \cup\langle S, \bar{S}\rangle_{-}$consisting of the forward cut edges $\langle S, \bar{S}\rangle_{+}$directed from $S$ to $\bar{S}$ and backward cut edges $\langle S, \bar{S}\rangle_{-}$directed from $\bar{S}$ to $S$. Let $G(S)$ be the subgraph induced on $S$ by removing $\langle S, \bar{S}\rangle$ from $G$. Similarly, let $G(\bar{S})$ be the subgraph induced on $\bar{S}$ by removing $\langle S, \bar{S}\rangle$ from $G$. If $G(S)$ and $G(\bar{S})$ are both connected, then $\langle S, \bar{S}\rangle$ is called a cutset of $G$. Let us assume $\langle S, \bar{S}\rangle$ is an arbitrary cut of $G$.

We define the enabling numbers of $G(S)$ and $G(\bar{S})$ as

$$
\mu(G(S)) \triangleq \min _{e \in\langle S, \bar{S}\rangle_{-}}\{M(e)\}
$$

and

$$
\mu(G(\bar{S})) \triangleq \min _{e \in\langle S, \bar{S}\rangle_{+}}\{M(e)\} .
$$

An elementary diakoptic firing of a vertex-induced subgraph $G(\cdot)$ of a marked graph $G$ is any legal firing sequence confined to vertices in $G(\cdot)$ which fires each vertex in $G(\cdot)$ exactly once. Note that this definition includes subgraphs $G(\cdot)$ consisting of disjoint components.

Theorem 4 (Diakoptic-Transition Theorem): An elementary diakoptic firing of a vertex-induced subgraph $G(S)$ of a marked graph $G$ is legal under a live marking $M$ if and only if $\mu(G(S))>0$.

Proof: First, we note that the markings of $G(S)$ and $G(\bar{S})$ are unaffected by the diakoptic firing of $G(S)$ since vertices in $\bar{S}$ are not fired and each vertex in $S$ is fired exactly once. The only edges of $G$ whose markings change in an elementary diakoptic firing of $G(S)$ are those of $\langle S, \bar{S}\rangle$. Each edge of $\langle S, \bar{S}\rangle_{\text {- loses one token and each }}$ 
edge of $\langle S, \bar{S}\rangle_{+}$gains one token. By hypothesis, $\mu(G(S))$ $>0$ and so, each edge $e \in\langle S, \bar{S}\rangle_{-}$has at least one token. Further, the edges of $\langle S, \bar{S}\rangle+$ play no role in determining the legality of a firing sequence confined to vertices in $S$. Hence, all edges in the cut $\langle S, \bar{S}\rangle$ may be removed from $G$, isolating $G(S)$ from $G(\bar{S})$ and so, we need only show that there exists a legal firing sequence of any marked graph $G$ from a live marking $M$ which fires each vertex of $G$ exactly once, returning to $M$. This question has already been resolved in [2]. However, we present an alternate proof which is easily extendable for the capacitated case.

Let $G\left(E_{f}\right)$ be the token-free subgraph of $G$ under $M$ induced by the token-free edge set $E_{f}=\{e \mid M(e)=0\}$.

Property 1: $G\left(E_{f}\right)$ is acyclic.

Property 2: A source in $G\left(E_{f}\right)$ is an enabled vertex in $G$ under $M$.

Property 1 follows from the liveness of $M$. That is, $G$ has no token-free directed circuits under $M$. Property 2 follows from the observation that a source of $G\left(E_{f}\right)$ is either a source of $G$ or a vertex of $G$ with at least one token on each edge incident into it under $M$. In either case, a source of $G\left(E_{f}\right)$ is an enabled vertex of $G$ under $M$. Since $G\left(E_{f}\right)$ is acyclic, it contains a source and hence, $G$ contains an enabled vertex $v$ under $M$. Firing this vertex $v$ results in a new marking of $G$ obtained by subtracting one token from each edge incident into $v$ and adding one token to each edge incident out of $v$. Since $v$ has been fired and each edge incident out of $v$ has at least one token then $v$ may be removed from $G$. The above argument recursively applies to the resulting subgraph of $G$ since it is another live marked graph for which an elementary diakoptic-firing sequence is sought.

As noted in the proof of Theorem 4, an elementary diakoptic firing of a subgraph $G(S)$ affects the marking of $G$ only on edges of the cut $\langle S, \bar{S}\rangle$. Specifically, one token is subtracted from all edges of $\langle S, \bar{S}\rangle$ incident into $G(S)$ and one token is added to all edges of $\langle S, \bar{S}\rangle$ incident out of $G(S)$. Thus we may view the state-transition process diakoptically. That is, we may consider $G(S)$ as a supernode of $G$. This is the essence of the above theorem. It tells us that we can move from marking to marking by firing supernodes or clusters at a time, ignoring the actual firing sequence involved within the cluster since the theorem guarantees its existence.

\subsection{A Diakoptic-Reachability Theorem}

The diakoptic-transition theorem for live-marked graphs provides us with a diakoptic-reachability theorem for such cases. The interesting aspect of this is that every reachable marking from an initial marking of a live-marked graph $G$ defines its own unique diakoptic firing sequence leading from the initial marking to that marking. We now proceed to develop this result.

Let $G=(V, E)$ be a marked graph on vertex set $V$, edge set $E$ and live initial marking $M_{0}$. Let $M_{f} \in R\left(M_{0}\right)$ and, as in [3], let $\Sigma_{0}=\left[\sigma_{1}^{0}, \sigma_{2}^{0} \cdots \sigma_{n}^{0}\right]^{t}$ be the minimum nonnegative solution to $A^{t} \Sigma=M_{f}-M_{0}$, where $A$ is the incidence matrix of $G$. Identify the entities defined by the following algorithm:

$$
\begin{aligned}
& \begin{array}{l}
k \leftarrow 0 \\
\text { While } \Sigma_{k} \triangleq\left[\sigma_{1}^{k}, \sigma_{2}^{k} \cdots \sigma_{n}^{k}\right]^{t} \neq 0 \text { do } \\
\text { begin } \\
S_{k} \leftarrow\left\{v \mid \sigma_{v}^{k}>0\right\} \\
G_{k} \leftarrow G\left(S_{k}\right) \\
x_{k} \leftarrow \min _{v \in S_{k}}\left\{\sigma_{v}^{k}\right\} \\
\sigma_{v}^{k+1} \leftarrow\left\{\begin{array}{l}
\sigma_{v}^{k}-x_{k}, \forall v \in S_{k} \\
0, \forall v \in \bar{S}_{k}
\end{array}\right. \\
k \leftarrow k+1
\end{array} \\
& \text { end }
\end{aligned}
$$

Theorem 5 (Diakoptic-Reachability Theorem): The diakoptic-firing sequence defined by the expression

$$
\prod_{k=0}^{r-1} G_{k}^{x_{k}}
$$

of length $r \leqslant n-1$ legally transforms $M_{0}$ into $M_{f}$ on $G$, where $G_{k}^{x_{k}}$ denotes $x_{k}$ successive elementary diakoptic firings of $G_{k}$.

Proof: First, let us recall that a diakoptic firing of a vertex-induced subgraph $G(S)$ of a marked graph $G$ affects the marking of $G$ on edges of the cut $\langle S, \bar{S}\rangle$ only. Also, for a live $G$, a diakoptic firing of $G(S)$ is legal if and only if $\mu(G(S))>0$. Thus we need only show that $x_{k} \leqslant$ $\mu\left(G_{k}\right)$, for $0 \leqslant k \leqslant r-1$. We demonstrate that $x_{0} \leqslant \mu\left(G_{0}\right)$ and then deduce that $x_{k} \leqslant \mu\left(G_{k}\right)$ for $1 \leqslant k \leqslant r-1$.

Let $\bar{S}_{k}=V-S_{k}$, for $0 \leqslant k \leqslant r-1$. Further, let $\left\langle S_{k}, \bar{S}_{k}\right\rangle_{+}$ and $\left\langle S_{k}, \bar{S}_{k}\right\rangle_{-}$denote the forward and backward edge sets of the cut $\left\langle\bar{S}_{k}, \bar{S}_{k}\right\rangle$, respectively, for $0 \leqslant k \leqslant r-1$, where "forward" is the direction from $S_{k}$ to $\bar{S}_{k}$. From the state equation, we have $M_{f}(e)=M_{0}(e)+\sigma_{i}^{0}-\sigma_{j}^{0}$, for each edge $e=(i, j) \in E$. Now, by definition, $\sigma_{i}^{0}=0, \forall i \in \bar{S}_{0}$. So, $M_{f}(e)=M_{0}(e)-\sigma_{j}^{0}, \forall e=(i, j) \in\left\langle S_{0}, \bar{S}_{0}\right\rangle_{-}$. Imposing nonnegativity on $M_{f}$, we obtain $\sigma_{j}^{0} \leqslant M_{0}(e), \forall e=(i, j) \in$ $\left\langle S_{0}, \bar{S}_{0}\right\rangle_{-}$. By definition, $x_{0} \leqslant \sigma_{j}^{0}, \forall j \in S_{0}$, and, thus it follows that $x_{0} \leqslant M_{0}(e), \forall e \in\left\langle S_{0}, \bar{S}_{0}\right\rangle_{-}$. The enabling number of $G_{0}$ is simply

$$
\mu\left(G_{0}\right)=\min _{e \in\left\langle S_{0}, \bar{S}_{0}\right\rangle_{-}}\left\{M_{0}(e)\right\}
$$

and it follows that $x_{0} \leqslant \mu\left(G_{0}\right)$. To deduce that the $(k+1)$ th diakoptic firing is legal given that the first $k$ diakoptic firings are legal, we need merely note that $\Sigma_{k}$ is the minimum nonnegative solution to $A^{t} \Sigma=M_{f}-M_{k}$, where $M_{k}$ is the marking of $G$ after the first $k$ diakoptic firings. Thus, the above argument is true when $S_{0}, x_{0}, M_{0}$, and $\Sigma_{0}$ are replaced with $S_{k}, x_{k}, M_{k}$, and $\Sigma_{k}$, respectively.

\subsection{Obtaining a Basic Marking in $R\left(M_{0}\right)$}

With the notion of a diakoptic transition established, we describe an algorithm for obtaining a basic $M \in R\left(M_{0}\right)$ when $M_{0}$ is live on $G$. We assume that $G$ is connected.

Suppose that, by some means, we have obtained an $M \in R\left(M_{0}\right)$ such that the subgraph $G(S)$, induced over some subset of vertices $S$, has a token-free spanning tree $T$ under $M$. Since $G$ is connected, $\langle S, \bar{S}\rangle \neq \varnothing$. Since $G$ is live 
under $M, G(S)$ is also live under $M$. If $\langle S, \bar{S}\rangle_{-} \neq \varnothing$ then fire $G(S)$ diakoptically $\mu=\mu(G(S))$ times. This transition is legal and results in a marking $M^{\prime}$ obtained from $M$ by subtracting $\mu$ tokens from each edge in $\langle S, \bar{S}\rangle_{-}$and adding $\mu$ tokens to each edge in $\langle S, \bar{S}\rangle_{+}$. If $\mu$ is not equal to zero, then at least one edge $e=(i, j) \in\langle S, \bar{S}\rangle_{-}$is token-free under $M^{\prime}$ and since the marking of $G(S)$ is unaffected by the diakoptic firing of $G(S)$, then $T$ is also token-free under $M^{\prime}$. Clearly, this is the case when $\mu=0$. Thus the tree $T \cup\{e\}$ is a token-free spanning tree of $G(S \cup\{i\})$ under $M^{\prime} \in R\left(M_{0}\right)$. If $\langle S, \bar{S}\rangle_{-}=\varnothing$ then $\langle S, \bar{S}\rangle_{+} \neq \varnothing$ and, so, by similar reasoning, we may fire $G(\bar{S})$, diakoptically, $\mu(G(\bar{S}))$ times. This transition is also legal and results in the marking $M^{\prime}$ obtained from $M$ by subtracting $\mu(G(\bar{S}))$ tokens from each edge in $\langle S, \bar{S}\rangle_{+}$. At least one edge $e=(i, j) \in\langle S, \bar{S}\rangle_{+}$is token-free under $M^{\prime}$ and so, $T \cup\{e\}$ is a token-free spanning-tree of $G(S \cup$ $\{j\})$ under $M^{\prime} \in R\left(M_{0}\right)$. Hence, we have an algorithm. We simply start with $S=\{v\}$ for any vertex $v \in G$ and with $T=\varnothing$.

The following algorithm will generate a basic marking $M$, reachable from a live marking $M_{0}$, for a connected marked graph $G$.

1) Set $T=\varnothing, M=M_{0}$ and $S=\{v\}$, for any $v \in V$.

2) While $|S| \leq|V|$ do

$$
\begin{aligned}
& \text { if }\langle S, \bar{S}\rangle_{-} \neq \varnothing \\
& \text { then } \\
& \text { begin }
\end{aligned}
$$

Compute $\mu \triangleq \mu(G(S))$ under $M$.

Fire $G(S) \mu$ times, updating $M$.

$T \leftarrow T \cup\{e\}$ and $S \leftarrow S \cup\{i\}$,

where $e=(i, j)$ is a token-free edge of $\langle S, \bar{S}\rangle_{-}$under $M$.

$$
\text { end }
$$$$
\langle S, \bar{S}\rangle_{-} \text {under } M \text {. }
$$

begin

Compute $\mu \triangleq \mu(G(\bar{S}))$ under $M$

Fire $G(\bar{S}) \mu$ times, updating $M$.

$T \leftarrow T \cup\{e\}$ and $S \leftarrow S \cup\{j\}$,

where $e=(i, j)$ is a token-free edge of $\langle S, \bar{S}\rangle_{+}$under $M$.

end

3) Stop. $M$ is a basic marking in $R\left(M_{0}\right)$ with the token-free spanning tree $T$.

We may modify this algorithm by including as many token-free edges as possible at each iteration, so long as a circuit does not form.

\subsection{Pivoting and Diakoptic Firing}

Next, let us interpret the fundamental operation of simplex, pivoting, in terms of vertex firing.

Each pivot of simplex selects one nonbasic variable (tree branch) with a corresponding positive relative cost (fundamental-cutset weight), and exchanges it with one basic variable (cospanning-tree chord). If no such exchange is possible (all relative branch costs are nonpositive) then we have an optimal marking. Let us examine the details of this branch-chord exchange.
Let $b=(u, v)$ be the branch of $T$ that has been selected to enter the basis (cospanning tree), where $b$ is incident out of vertex $u$ and into vertex $v$. Now, breaking the branch $b$ splits $T$ into exactly two fragments, $T_{u}$ and $T_{v}$, where $u \in T_{u}$ and $v \in T_{v}$. Let $S$ be the set of vertices that $T_{u}$ spans and then $T_{v}$ spans $\bar{S}=V-S$. The fundamental cutset of $G$ that $b$ defines is simply $\langle S, \bar{S}\rangle$. Thus since $b$ has been selected to leave the tree and enter the cotree, then $W(\langle S, \bar{S}\rangle)>0$ because the relative cost of branch $b$ is the weight of $\langle S, \bar{S}\rangle$.

Let this first pivot move the state from $M_{0}$ to, say, $M_{1}$. Simplex moves from one basic feasible solution to another basic feasible solution with each pivot. Thus for $M_{1}$ to be basic, it must also possess a token-free spanning tree $T_{1}$. To ensure $M_{1}$ is a basic marking, we must select a chord from $\bar{T}$ so as to reconnect the fragments $T_{u}$ and $T_{v}$. The only edges of $G$ which connect vertices in $T_{u}$ to vertices in $T_{v}$ are edges of $\langle S, \bar{S}\rangle$. Hence, we must exchange $b$ with one of the chords in its fundamental cutset. When the exchange is made, the cutset associated with the new tree branch is the same cutset associated with $b$ in the original tree, but its orientation is now defined by the new branch. Now, we must determine a selection rule and the update procedure.

This is where the diakoptic property of the state-transition process is useful in explaining the pivot operation. Let $\mu=\mu(G(S))$. We know that $G(S)$ can be legally, diakoptically fired $\mu$ times and this subtracts $\mu$ tokens from each edge of $\langle S, \bar{S}\rangle_{-}$and adds $\mu$ tokens to each edge of $\langle S, \bar{S}\rangle_{+}$. This operation increases the objective function $W M$ by an amount $\mu W(\langle S, \bar{S}\rangle)$. This is exactly the pivot operation of simplex. If $\mu=0$ then we are experiencing degeneracy.

Now, at least one edge $d \in\langle S, \bar{S}\rangle_{\text {- }}$ is token-free under $M_{1}$. If there are more, then each token-free edge in $\langle S, \bar{S}\rangle_{\text {- }}$ is a candidate for leaving the basis. Let us disregard the possibility of more than one leaving candidate, for now, by assuming that $d$ is unique in each pivot. Then, the basis exchange is denoted by the expressions $\bar{T}_{1}=\bar{T}+\{b\}-\{d\}$ and $T_{1}=T-\{b\}+\{d\}$.

Instead of computing the relative branch costs associated with the branches of $T_{1}$ from scratch, we would rather have a method that transforms the relative cost vector of $T$ into the relative branch costs of $T_{1}$. This could be computationally efficient if the relative costs of $T_{1}$ can be obtained from those of $T$ through a reasonably simple transformation. This looks like a fruitful approach since $T$ and $T_{1}$ differ in only two edges. Indeed, this is the case, as the next theorem illustrates.

Since the relative branch costs are the corresponding fundamental-cutset weights, we must transform the fundamental-cutset weights of $T$ into those of $T_{1}$. Now, $T+\{d\}$ contains exactly one circuit $C$, whose orientation is defined by the orientation of chord $d$. The branch $b$ is a member of $C$ and follows the circuit direction. It can be easily seen that the only fundamental cutsets affected by the transformation

$$
T_{1}=T+\{d\}-\{b\}
$$


are those associated with edges of $C$ (think of the column associated with $d$ in $Q_{f}$ ). Therefore, we need only update the relative costs associated with $C$, in moving from $T$ to $T_{1}$.

Theorem 6: The relative-cost vector $\tilde{W}_{1}$ of $T_{1}=T-\{b\}$ $+\{d\}$ is equal to the relative-cost vector $\tilde{W}$ of $T$ minus $W(\langle\mathrm{~S}, \bar{S}\rangle)$ on all forward edges of $C$ plus $W(\langle S, \bar{S}\rangle)$ on all backward edges of $C$.

Proof: The result is true for edges $b$ and $d$ since they are in the same direction with respect to $C$ and the relative costs of both $b$ and $d$ decrease by $W(\langle S, \bar{S}\rangle)$ when they exchange roles. Let $(x, y)$ be an edge of $C$ other than $b$ and $d$, let $\left\langle S_{x}, \bar{S}_{x}\right\rangle$ denote its fundamental cutset in $T$ and let $\left\langle S_{x}^{1}, \bar{S}_{x}^{1}\right\rangle$ denote its fundamental cutset in $T_{1}$. There are four cases to consider.

Case 1: $(x, y) \in T_{v}$ and follows the orientation of $C$.

In this case, $S \subset S_{x}$ and $S_{x}^{1}=S_{x}-S$. Therefore, we may write the weight of the new cutset as

$$
\begin{aligned}
W\left(\left\langle S_{x}^{1}, \bar{S}_{x}^{1}\right\rangle\right)= & W\left(\left\langle S_{x}-S,\left(V-S_{x}\right) \cup S\right\rangle\right) \\
= & W\left(\left\langle S_{x}-S, \dot{V}-S_{x}\right\rangle\right)+W\left(\left\langle S_{x}-S, S\right\rangle\right) \\
= & W\left(\left\langle S_{x}, V-S_{x}\right\rangle\right)-W\left(\left\langle S, V-S_{x}\right\rangle\right) \\
& -W\left(\left\langle S, S_{x}-S\right\rangle\right) \\
= & W\left(\left\langle S_{x}, \bar{S}_{x}\right\rangle\right)-W(\langle S, \bar{S}\rangle) .
\end{aligned}
$$

Case 2: $(x, y) \in T_{v}$ and opposes the orientation of $C$. In this case, $S_{x} \subset \bar{S}$ and $S_{x}^{1}=S_{x} \cup S$. Clearly, this will lead to

$$
W\left(\left\langle S_{x}^{1}, \bar{S}_{x}^{1}\right\rangle\right)=W\left(\left\langle S_{x}, \bar{S}_{x}\right\rangle\right)+W(\langle S, \bar{S}\rangle)
$$

Case 3: $(x, y) \in T_{u}$ and follows the orientation of $C$. Here $S_{x} \subset S$ and $S_{x}^{1}=S_{x} \cup \bar{S}$, which will yield

$$
\begin{aligned}
W\left(\left\langle S_{x}^{1}, \bar{S}_{x}^{1}\right\rangle\right) & =W\left(\left\langle S_{x}, \bar{S}_{x}\right\rangle\right)+W(\langle\bar{S}, S\rangle) \\
& =W\left(\left\langle S_{x}, \bar{S}_{x}\right\rangle\right)-W(\langle S, \bar{S}\rangle) .
\end{aligned}
$$

Case 4: $(x, y) \in T_{u}$ and opposes the orientation of $C$.

Now, we have $\bar{S} \subset S_{x}$ and $S_{x}^{1}=S_{x}-\bar{S}$, which leads to the expression

$$
W\left(\left\langle S_{x}^{1}, \bar{S}_{x}^{1}\right\rangle\right)=W\left(\left\langle S_{x}, \bar{S}_{x}\right\rangle\right)+W(\langle S, \bar{S}\rangle) .
$$

This theorem implies that we may treat the relative costs as currents or flows, because, as we move from tree to tree or more appropriately from one cospanning tree to another, the relative costs always satisfy the same nodal equations. Thus we may now state the basic step of our algorithm. First, compute the $n-1$ fundamental-cutset weights by inspection, and establish a current $I_{0}(b)$ on each branch $b$ of the initial spanning tree $T_{0}=T$, equal to its corresponding fundamental-cutset weight $W(\langle S, \bar{S}\rangle)$. Set $I_{0}(d)=0$, for all chords of the initial cospanning tree $\bar{T}_{0}=T$. Assuming degeneracy is not present, then the $k$ th basic step of the algorithm is

A: Locate a branch $b \in T_{k}$ with a positive current $I_{k}(b)$. If no such branch exists, then $M_{k}$ is optimal;

B: Compute $\mu=\mu(G(S))$ under $M_{k}$;

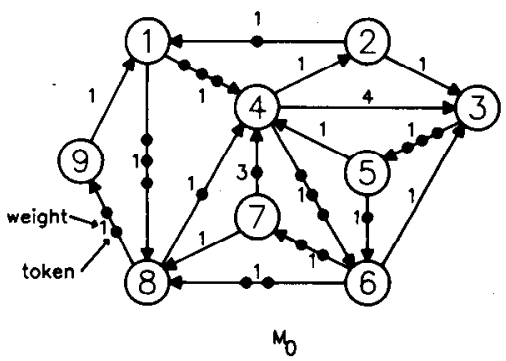

(a)

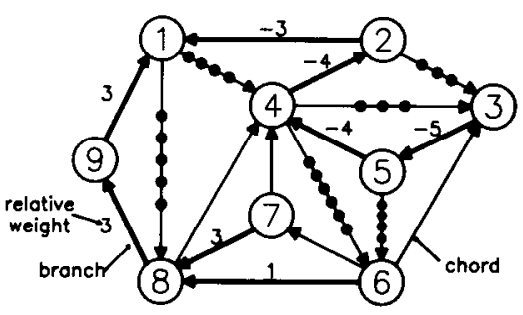

$M_{1}$

(b)

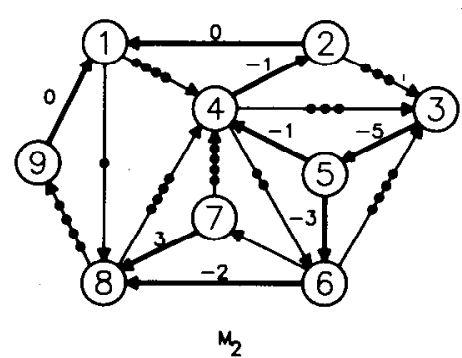

(c)

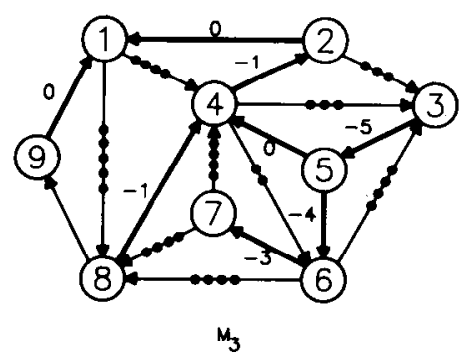

(d)

Fig. 1 .

C: Fire $G(S), \mu$ times. That is, subtract $\mu$ tokens from all backward edges of $\langle S, \bar{S}\rangle$ and add $\mu$ tokens to all forward edges of $\langle S, \bar{S}\rangle$, to yield $M_{k+1}$;

D: Augment the current flowing in $C$. That is, subtract $I_{k}(b)$ flow units from all forward edges of $C$ and add $I_{k}(b)$ flow units to all backward edges of $C$ to yield $I_{k+1}$;

E: Let $\bar{T}_{k+1}=\bar{T}_{k}+\{b\}-\{d\}, T_{k+1}=T_{k}-\{b\}+\{d\}$.

As an example, to illustrate our maximum-weight marking algorithm, consider the marked graph with marking $M_{0}$ shown in Fig. 1(a). In Fig. 1(b) is shown a basic marking $M_{1}$ reachable from $M_{0} . M_{1}$ is obtained from $M_{0}$ through the sequence of diakoptic firings $G_{1}^{0} G_{2}^{1} G_{3}^{0} G_{4}^{0} G_{5}^{1} G_{6}^{0} G_{7}^{2} G_{8}^{0}$, where the subgraphs are induced on 
the vertex sets

$$
\begin{aligned}
& S_{1}=\{1\} \\
& S_{2}=\{1,9\} \\
& S_{3}=\{1,2,9\} \\
& S_{4}=\{1,2,4,9\} \\
& S_{5}=\{1,2,4,5,9\} \\
& S_{6}=\{1,2,4,5,8,9\} \\
& S_{7}=\{1,2,4,5,7,8,9\} \\
& S_{8}=\{1,2,3,4,5,7,8,9\}
\end{aligned}
$$

respectively.

The relative costs associated with the branches defining $M_{1}$ are as shown in Fig. 1(b). Note the relative costs for the chords are all zero.

It can be seen that the relative cost for the branch $(8,9)$ is greater than zero. One chord $(5,6)$ in the fundamental cutset of this branch has a minimum marking. Thus we have to exchange $(8,9)$ with $(5,6)$. This requires firing the subgraph on the vertex set $\{6,7,8\} 4$ times and we get the new basic marking $M_{2}$ shown in Fig. 1(c) which may be seen to be a degenerate marking. Updating the relative costs as described in Section 3.6, we get the' relative costs of the branches defined by $M_{2}$ as shown in Fig. 1(c).

Continuing the algorithm, we obtain the maximum-weight marking $M_{3}$ shown in Fig. 1(d). This. is achieved through the sequence of diakoptic firings $G^{0}(\{7\}) G^{4}(\{1,2,3,4,5,6,7,9\})$. It may be noted from Fig. 1(d) that all of the relative costs are nonpositive indicating that $M_{3}$ is an optimum marking. The weight of this optimum marking is 50 .

\subsection{Boundedness}

The conditions governing boundedness follow easily.

Suppose that simplex encounters a directed cutset $\langle S, \bar{S}\rangle$, defined by branch $b$ of spanning tree $T$, at some pivot, for some instance of a maximum-weight marking problem. Then the enabling number of $G(S)$ is undefined. $G(S)$ is a diakoptic source. That is, $G(S)$ can be diakoptically fired an indefinite number of times. Every diakoptic firing of $G(S)$ increases the marking on each edge of $\langle S, \bar{S}\rangle$. Since $b$ has been selected to enter the basis $W(\langle S, \bar{S}\rangle)>0$. Thus, the problem is unbounded. Boundedness of the maximum-weight marking problem is, thus characterized in the following theorem.

Theorem 7: The weight $W M$ of a marking is bounded on $R\left(M_{0}\right)$ if and only if $G$ does not contain a positiveweight directed cutset.

Note that the boundedness condition given in [2], for the marking $M$ of a marked graph, follows as a special case of Theorem 7 when $W=[1,1, \cdots, 1]$. Thus we have the following corollary.

Corollary 7.1: The marking of a marked graph $G$ is bounded over $R\left(M_{0}\right)$ if and only if $G$ is strongly connected.

\section{MAXIMUM-Weight MARKING FOR A Capacitated Marked Graph}

The results of the previous sections are now extended to cover capacitated marked graphs.

We define a capacitated marked graph as a marked graph $G=(V, E)$ with the following extension. For each edge $e \in E$, a lower bound $L(e)$ and upper bound $U(e)$ are specified on the token count $M(e)$.

The introduction of lower bounds puts the problem in its most general form and does not complicate matters significantly. The definition reduces to the usual definition when $L(e)=0$ and $U(e)=\infty$ for all $e \in E$. We make the usual consistency assumption $L(e) \leqslant U(e), \forall e \in E$ which we denote in vector format as $L \leqslant U$. We make the further assumption $L<U$ since if $L(e)=U(e)$ for some edge $e=(i, j) \in E$ then vertices $i$ and $j$ are dead in every marking of $G$. In other words, the vertices $i$ and $j$ are not enabled under any marking reachable from $M_{0}$.

As before, let us relax the dead-circuit condition in the reachability theorem by considering live problems only. Then, for capacitated marked graphs, Program (1) is equivalent to the linear program

$$
\begin{aligned}
\operatorname{maximize} W M & \\
\text { subject to } & B_{f} M=Z_{\bar{T}} \\
& L \leqslant M \leqslant U .
\end{aligned}
$$

We need only outline the extensions from this point.

\subsection{Basic Markings}

A cospanning tree still constitutes a basis since the equation constraints are circuit equations of $G$. However, we must modify our definition of a basic marking. To this end, let $M \in R\left(M_{0}\right)$. An edge $e \in E$ is called depleted under the marking $M$ if $M(e)=L(e)$. Similarly, an edge $e \in E$ is saturated under $M$ if $M(e)=U(e)$. So, we define a basic marking in these terms.

A marking $M$ of $G$ is called a basic marking if there exists a spanning tree of $G$ composed solely of branches that are either depleted or saturated under $M$.

\subsection{Diakoptic Transitions}

We must establish a diakoptic-transition theorem for the class of live problems on capacitated-marked graphs. We define the enabling number of a vertex induced subgraph $G(S)$ of $G$ under $M$ as

$$
\begin{array}{r}
\mu(G(S)) \triangleq \min \left\{\min _{e \in\langle S, \bar{s}\rangle_{-}}\{M(e)-L(e)\},\right. \\
\left.\min _{e \in\langle S, \bar{s}\rangle_{+}}\{U(e)-M(e)\}\right\}
\end{array}
$$

which reduces to the usual definition when $L(e)=0$ and $U(e)=\infty$, for all $e \in E$.

Theorem 8: An elementary diakoptic firing of $G(S)$ is legal if and only if $\mu(G(s))>0$.

Proof: Again, the proof follows from the reachability thcorcm for capacitated marked graphs [6]. However, we can provide a constructive procedure similar to that in 
the proof of Theorem 4. As in the proof of Theorem 4, we need only prove that there exists a legal firing sequence of any capacitated marked graph $G$ from a live marking $M$ which fires each vertex of $G$ exactly once, returning to $M$.

Construct a graph $G^{\prime}$ from $G$ by open-circuiting all edges of $G$ which are neither depleted nor saturated under $M$ and reversing the direction of all saturated edges under $M$.

Property 1: $G^{\prime}$ is acyclic.

Property 2: A source in $G^{\prime}$ is an enabled vertex in $G$ under $M$.

Property 1 follows from the liveness assumption since, by definition, a dead-circuit in $G$ under $M$ is represented by a directed circuit in $G^{\prime}$. Property 2 follows from the observation that any source of $G^{\prime}$ is a vertex of $G$ with no depleted input edges and no saturated output edges under $M$. Properties 1 and 2 imply that $G$ has an enabled vertex $v$ under $M$. After vertex $v$ fires, it has no depleted output edges and no saturated input edges and hence, it may be removed from $G$ since the edges incident on it do not restrict the remaining sequence. As in the proof of Theorem 4 , the above argument applies to the remaining subgraph and proof is established by recursion.

\subsection{Obtaining a Basic Marking in $R\left(M_{0}\right)$}

We show, by construction, that it is possible to obtain a basic marking $M \in R\left(M_{0}\right)$, as defined for a capacitated marked graph, using the notion of a diakoptic transition.

If $G$ is connected and $U$ is finite then it is clear from the definition that the enabling number of any subgraph $G(S)$ of $G$ is always defined. However, to present the result in its most general form, we assume that $U$ may have infinite entries.

To simplify the algorithm description, we define the input and output enabling numbers of a subgraph $G(S)$ under $M$ as

$\mu_{i}(G(S)) \triangleq \begin{cases}\infty, & \text { if }\langle S, \bar{S}\rangle_{-}=\varnothing \\ \min _{e \in\langle S, \bar{S}\rangle_{-}}\{M(e)-L(e)\}, & \text { otherwise }\end{cases}$

and

$\mu_{o}(G(S)) \triangleq \begin{cases}\infty, & \text { if }\langle S, \bar{S}\rangle_{+}=\varnothing \\ \min _{e \in\langle S, \bar{S}\rangle_{+}}\{U(e)-M(e)\}, & \text { otherwise }\end{cases}$

respectively. Then, $\mu(G(S)) \triangleq \min \left\{\mu_{i}(G(S)), \mu_{0}(G(S))\right\}$. Using these definitions, the following algorithm constructs a basic marking $M \in R\left(M_{0}\right)$, for a capacitated marked graph $G=(V, E)$.

1). Set $M=M_{0}, T=\varnothing$ and $S=\{v\}$ for any $v \in V$.

2) While $|S|<|V|$ do

\section{begin}

$$
\begin{aligned}
& \mu_{i} \leftarrow \mu_{i}(G(S)), \mu_{o} \leftarrow \mu_{o}(G(S)) \text { and } \\
& \mu \leftarrow \min \left\{\mu_{i}, \mu_{o}\right\} \text { under } M \\
& \text { if } \mu<\infty \\
& \text { then } \\
& \quad \text { begin }
\end{aligned}
$$

Fire $G(S) \mu$ times, updating $M$.

if $\mu_{i}<\mu_{0}$ then $T \leftarrow T \cup\{e\}$ and $S \leftarrow S \cup\{i\}$, where $e=(i, j) \in\langle S, \bar{S}\rangle_{\text {- with }}$ $M(e)=L(e)$. else $T \leftarrow T \cup\{e\}$ and $S \leftarrow S \cup\{j\}$, where $e=(i, j) \in\langle S, \bar{S}\rangle_{+}$with $M(e)=U(e)$.

end

begin

$\mu \leftarrow \mu(G(\bar{S}))$ under $M$.

Fire $G(\bar{S}) \mu$ times, updating $M$.

$T \leftarrow T \cup\{e\}$ and $S \leftarrow S \cup\{j\}$, where $e=(i, j) \in\langle S, \bar{S}\rangle_{+}$with $M(e)=L(e)$.

end

else

end

3) Stop. $M$ is a basic marking in $R\left(M_{0}\right)$ with spanning tree $T$.

\subsection{Conditions for Optimality}

As usual, $\langle S, \bar{S}\rangle$ denotes the fundamental cutset defined by branch $b \in T$. Optimality is characterized in the following definition.

A basic marking $M \in R\left(M_{0}\right)$ with basis $\bar{T}$ is maximum over $R\left(M_{0}\right)$ if and only if $W(\langle S, \bar{S}\rangle) \geqslant 0$ for every saturated branch $b=(i, j) \in T$ and $W(\langle S, \bar{S}\rangle) \leqslant 0$ for every depleted branch $b \in T$, where $i \in S$ and $j \in \bar{S}$.

To establish that this is indeed a sufficient condition for optimality of a basic marking $M \in R\left(M_{0}\right)$, we need only substitute the corresponding basic dictionary into the objective from which we conclude that the objective cannot be increased by effecting a change in the marking $M(b)$ of any branch $b \in T$ and that the current value of the objective is an upperbound on $W M$ over $R\left(M_{0}\right)$. Hence such a basic marking is optimal.

\subsection{Pivoting and Diakoptic Firing}

We may restate the optimality condition as follows.

A basic marking $M \in R\left(M_{0}\right)$ is maximum over $R\left(M_{0}\right)$ if and only if $T$ contains no depleted branch $b=(i, j)$ with $W(\langle S, \bar{S}\rangle)>0$ and no saturated branch $b=(i, j)$ with $W(\langle S, \bar{S}\rangle)<0$, where $i \in S$ and $j \in \bar{S}$.

Hence, we may use the method described in Section 3.6 to achieve an optimal basic marking with some slight modifications. From the above condition, a branch $b \in T$ is a candidate for entering the basis if it is depleted and $W(\langle S, \bar{S}\rangle)>0$ or if it is saturated and $W(\langle S, \bar{S}\rangle)<0$.

Now, if the entering branch $b$ is depleted, then $W(\langle S, \bar{S}\rangle)>0$ indicates that firing $G(S)$ will increase the objective. Similarly, for a saturated branch $b, W(\langle S, \bar{S}\rangle)$ $<0$ indicates that firing $G(\bar{S})$ will increase the objective. Hence, the pivot operation follows. If the entering branch $b$ is depleted then $G(S)$ is fired $\mu(G(S))$ times. Otherwise, $G(\bar{S})$ is fired $\mu(G(\bar{S}))$ times. The progress achieved in the pivot is $\mu(G(S)) W(\langle S, \bar{S}\rangle) \geqslant 0$ if $b$ is depleted or $\mu(G(\bar{S})) W(\langle\bar{S}, S\rangle) \geqslant 0$ if $b$ is saturated. 
The basis exchange is easily seen. The greedy diakoptic firing of $G(S)$ or $G(\bar{S})$ either depletes or saturates at least one chord $d \in\langle S, \bar{S}\rangle$. Each depleted or saturated chord $d \in\langle S, \bar{S}\rangle$, after a pivot, is a candidate for leaving the basis. In general, there may be multiple depleted chords and/or multiple saturated chords competing for the leaving variable. This represents a degenerate basis and we cannot simply ignore the multiplicity by selecting any candidate at random, as this can lead to cycling in the algorithm. However, since the details of degeneracy are subtle, we shall assume that simplex will not encounter a degenerate basis and note that there exists a number of anticycling techniques which are applicable to general $L P$ problems. Hence, the assumption uniquely specifies a chord $d \in\langle S, \bar{S}\rangle$ which is either depleted or saturated after the pivot. Again, the basis exchange is denoted as $\bar{T} \leftarrow \bar{T}+\{b\}$ $-\{d\}$, or equivalently, $T \leftarrow T-\{b\}+\{d\}$.

The relative-cost coefficients are updated in exactly the same manner as in the uncapacitated case. This is intuitively obvious since the capacities restrict the markings of $G$ and are in no relation with the wcights.

\subsection{Boundedness}

The boundedness condition follows from arguments similar to those used in the uncapacitated case and is summarized in the following theorem.

Theorem 9: The objective $W M$ is bounded over $R\left(M_{0}\right)$ if and only if $G$ contains no positive-weight directed cutset $\langle S, \bar{S}\rangle$ with $U(e)=\infty$ for all $e \in\langle S, \bar{S}\rangle$.

\subsection{Alternative Formulation of the Problem}

We now present an alternative formulation of the maximum-weight marking problem, which is suitable for studying the nonlive case.

Let $\omega_{i} \triangleq W(\langle\{i\}, V-\{i\}\rangle)$ denote the weight of vertex $i \in V$. The row vector of vertex weights $\Omega=\left[\omega_{i}\right]$ is then

$$
\Omega \triangleq W A^{t}
$$

where $A$ is the incidence matrix of $G$. The number $\omega_{i}$ represents the gain achieved in the objective $W M$ each time vertex $i$ is fired. Thus if the initial marking $M_{0}$ has an objective value $J_{0}=W M_{0}$ and vertex $i$ is fired $\sigma_{i}$ times, resulting in a marking $M$ with an objective value $J=W M$, then the number $\omega_{i} \sigma_{i}$ is the increase, $J-J_{0}$, achieved in the objective, in moving from $M_{0}$ to $M$. Since $J_{0}$ is fixed, then maximizing $J-J_{0}$ is equivalent to maximizing $J$. In fact, multiplying the state equation by $W$ gives

$$
W M=W M_{0}+\Omega \Sigma,
$$

where $\Sigma$ is the firing-count vector realizing $M$ from $M_{0}$. Hence, we may replace the objective $W M$ with the equivalent relative objective $\Omega \Sigma$.

Using the state equation, $M=M_{0}+A^{t} \Sigma$, we may pose the feasibility condition for a marking $M$ of $G$ in terms of the firing numbers. That is, $L \leqslant M \leqslant U$ means $L \leqslant M_{0}+$ $A^{t} \Sigma \leqslant U$. Thus the maximum-weight marking problem may be stated with the alternative linear program

$$
\begin{aligned}
& \text { maximize } \Omega \Sigma \\
& \text { subject to } L-M_{0} \leqslant A^{t} \Sigma \leqslant U-M_{0} .
\end{aligned}
$$

The special case of Program 4 when $L(e)=0$ and $U(e)=\infty$, for all $e \in E$, namely.

$$
\begin{aligned}
& \text { maximize } \Omega \Sigma \\
& \text { subject to } A^{t} \Sigma \geqslant-M_{0}
\end{aligned}
$$

represents the uncapacitated problem.

Note that $\Sigma$ is not explicitly restricted in either of these programs and that for any solution $\Sigma$, we can obtain the minimum nonnegative solution $\Sigma_{0}$, as in [3].

\section{MaXimum-Weight Markings for Nonlive MARKED GRAPHS}

It should be easy to see that the dead-circuit condition in the reachability theorem can be incorporated into the alternative formulation of the maximum-weight marking problem as follows. Let $D$ be the set of all vertices in $G$ belonging to a dead-circuit at $M_{0}$. By definition, no vertex in $D$ can fire at any $M \in R\left(M_{0}\right)$. Thus we need merely fix the firing count of each vertex $i \in D$ at zero. Then, for any marked graph $G$, the maximum-weight marking problem may be stated as

$$
\begin{aligned}
\operatorname{maximize} \Omega \Sigma & \\
\text { subject to } L-M_{0} & \leqslant A^{t} \Sigma \leqslant U-M_{0} \\
\sigma_{i} & =0, \quad \forall i \in D .
\end{aligned}
$$

The above program can be simplified as follows. Consider the subgraph $G(D)$ of $G$. Since each vertex $i \in D$ is dead then the marking of $G(D)$ cannot change in any legal firing sequence of $G$ from $M_{0}$. That is, $M(e)=M_{0}(e)$, $\forall e \in G(D), \forall M \in R\left(M_{0}\right)$. Thus all vertices in $D$ may be short-circuited together into a single dead vertex $d$ and then the self-loops induced on vertex $d$ may be removed. To incorporate the dead-circuit condition, we simply fix $\sigma_{d}=0$ and solve the problem for the reduced graph. The set $D$ is easy to identify.

\section{Structure of the Alternate Formulation}

Program 5 is formulated in terms of the firing counts and not in terms of markings as in the previous sections. We now demonstrate that the maximum-weight marking problem for a nonlive marked graph can be formulated as an auxiliary capacitated maximum-weight marking problem and that the methods discussed thus far are applicable to nonlive problems as well.

To simplify our presentation, we consider only the maximum-weight marking problem for nonlive uncapacitated marked graphs. The capacitated case can be studied in a similar way. The linear program form of this problem is the special case of Program 5, namely,

maximize $\Omega \Sigma$

$$
\begin{aligned}
\text { subject to } A^{t} \sum & \geqslant-M_{0} \\
\sigma_{i} & =0 \quad \forall i \in D .
\end{aligned}
$$


The first step in solving this problem with simplex is to convert the inequality constraints to equality constraints by introducing slack variables. It is easy to see that the column vector of slack variables for Program 6 is the final marking $M$ that $\Sigma$ realizes from $M_{0}$. Thus incorporating slack variables in Program 6 yields the equivalent program

maximize $\Omega \Sigma$

$$
\text { subject to } \begin{aligned}
M-A^{t} \Sigma & =M_{0} \\
\sigma_{i} & =0, \quad \forall \in D .
\end{aligned}
$$

Using the unit or identity matrix $I_{m}$ of dimension $m$, we may then organize Program 7 as

maximize $\Omega \Sigma$

$$
\begin{aligned}
\text { subject to }\left[I_{m}-A^{t}\right]\left[\begin{array}{c}
M \\
\Sigma
\end{array}\right] & =M_{0} \\
\sigma_{i} & =0, \quad \forall i \in D .
\end{aligned}
$$

Now, construct an auxiliary graph $\hat{G}$ from the original graph $G$ by introducing an artificial reference vertex external to $G$ and then connecting each vertex in $G$ to this reference vertex with an artificial edge. Formally, define the auxiliary graph $\hat{G}=(\hat{V}, \hat{E})$ for the original graph $G=$ $(V, E)$ according to

$$
\begin{aligned}
& \hat{V} \triangleq V \cup\{r\} \\
& \hat{E} \triangleq E \cup T^{*}
\end{aligned}
$$

where $r$ is an artificial reference vertex external to $G$ and

$$
T^{*} \triangleq\{e \mid e=(i, r), i \in V\}
$$

is a star tree consisting of artificial edges directed into the artificial reference. In fact, $T^{*}$ is a spanning tree of $\hat{G}$, and consequently, $E$ is a cospanning tree of $\hat{G}$. Hence, each edge of $G$ defines a fundamental circuit of $\hat{G}$. Let $\hat{B}_{f}$ be the fundamental-circuit matrix of $\hat{G}$ defined by its cospanning tree $E$. Then, the canonical form of $\hat{B}_{f}$ is $\left[I_{m} \hat{B}_{f n}\right]$. It is easy to see that

$$
\hat{B}_{f n}=-A^{t}
$$

and hence, $\left[I_{m}-A^{t}\right]$ is the fundamental-circuit matrix $\hat{B}_{f}$ of $\hat{G}$ with respect to the tree/cotree partition $\left(T^{*}, E\right)$ of $\hat{G}$. We may construct an auxiliary problem for any instance of Program 6 as follows.

Define an initial marking $\hat{M}_{0}$ of $\hat{G}$ according to

$$
\hat{M}_{0}(e) \triangleq \begin{cases}M_{0}(e) & \text { if } e \in E \\ 0 & \text { if } e \in T^{*}\end{cases}
$$

or simply,

$$
\begin{aligned}
& \hat{M}_{0}(E) \triangleq M_{0} \\
& \hat{M}_{0}\left(T^{*}\right) \triangleq 0
\end{aligned}
$$

where $\hat{M}_{0}(E)$ is the restriction of $\hat{M}_{0}$ to $E$. Note that $\hat{M}_{0}$ is a basic marking of $\hat{G}$ with basis $E$ and cobasis $T^{*}$.

Define an upperbound $\hat{U}$ on the marking $\hat{M}$ of $\hat{G}$ according to

$$
\hat{U}(e) \triangleq \begin{cases}\infty & \text { if } e \in E \\ u_{i} & \text { if } e \in T^{*} \text { and } e=(i, r)\end{cases}
$$

where

$$
u_{i} \triangleq \begin{cases}0, & \text { if } i \in D \\ \infty, & \text { if } i \in \bar{D} \triangleq V-D .\end{cases}
$$

Define a weighting $\hat{W}$ of $\hat{G}$ as

$$
\hat{W}(e) \triangleq \begin{cases}0, & \text { if } e \in E \\ \omega_{i} & \text { if } e \in T^{*} \text { and } e=(i, r)\end{cases}
$$

or simply,

$$
\begin{gathered}
\hat{W}(E) \triangleq 0 \\
\hat{W}\left(T^{*}\right) \triangleq \Omega .
\end{gathered}
$$

We define the auxiliary program associated with Program 6 as

$$
\begin{array}{r}
\operatorname{maximize} \hat{W} \hat{M} \\
\text { subject to } \hat{B}_{f} \hat{M}=M_{0} \\
0 \leqslant \hat{M} \leqslant U
\end{array}
$$

where $\hat{M}$ is a marking of $\hat{G}$.

We proceed to demonstrate that Programs 8 and 9 are equivalent.

Let $\hat{R}\left(\hat{M}_{0}\right)$ denote the solution space of the constraints in Program 9 and, as usual, let $R\left(M_{0}\right)$ denote the reachability set of $M_{0}$ on $G$.

Consider any $\hat{M} \in \hat{R}\left(M_{0}\right)$. Clearly, $\hat{M}$ is a feasible solution to Program 9. Let $\hat{\Sigma} \triangleq\left[\hat{\sigma}_{1}, \hat{\sigma}_{2}, \cdots, \hat{\sigma}_{n}, \hat{\sigma}_{r}\right]^{t}$ be any solution to $\hat{A}^{t} \hat{\Sigma}=\hat{M}-\hat{M}_{0}$, where $\hat{A}$ is the incidence matrix of $\hat{G}$. We have $\hat{M}(e)=\hat{M}_{0}(e)+\hat{\sigma}_{i}-\hat{\sigma}_{r}, \forall e \in T^{*}$. Since $\hat{M}_{0}(e)=0, \forall e \in T^{*}$, then $\hat{M}(e)=\hat{\sigma}_{i}-\hat{\sigma}_{r}, \forall e \in T^{*}$. Imposing nonnegativity on $\hat{M}$ yields $\hat{\sigma}_{i}-\hat{\sigma}_{r} \geqslant 0, \forall e \in T^{*}$ or $\hat{\sigma}_{r} \leqslant \hat{\sigma}_{i}, \forall i \in V$. Hence, $\hat{\sigma}_{r}^{0}=0$ in the minimum nonnegative solution $\hat{\Sigma}_{0}=\left[\hat{\sigma}_{1}-\hat{\sigma}_{r}, \hat{\sigma}_{2}-\hat{\sigma}_{r}, \cdots, \hat{\sigma}_{n}-\hat{\sigma}_{r}, 0\right]^{t}$ of $\hat{A}^{t} \hat{\Sigma}=\hat{M}$ $-\hat{M}_{0}$ and $\hat{\sigma}_{i}^{0}=\hat{M}(e), \forall e \in T^{*}$. Since $\hat{M} \leqslant U$, then $\hat{\sigma}_{i}^{0} \leqslant u_{i}$, $\forall i \in V$ or $\hat{\sigma}_{i}^{0}=0, \forall i \in D$.

If we let

$$
\Sigma_{0} \triangleq\left[\hat{\sigma}_{1}^{0}, \hat{\sigma}_{2}^{0}, \cdots, \hat{\sigma}_{n}^{0}\right]^{t}
$$

then we can see that

$$
A^{t} \Sigma_{0}=\hat{M}(E)-\hat{M}_{0}(E)=\hat{M}(E)-M_{0} .
$$

This means that $M=\hat{M}(E)$ and $\Sigma_{0}$ as defined in (10) constitutes a feasible solution of Program 8. Furthermore, this solution of (8) corresponding to $\hat{M}$ is unique since $\hat{\sigma}_{i}^{0}=0$ for at least one value of $i \in V$. Thus it follows that each feasible solution $\hat{M}$ of (9) defines a unique feasible solution of (8). Further, the objective values corresponding to these solutions are both equal to $\Omega \Sigma_{0}$. And, $\Omega \Sigma_{0}=\hat{W} \hat{M}$.

Starting from any feasible solution of (8) and retracing the above arguments, we can show that each such feasible solution defines a unique feasible solution of (9), and the corresponding objectives are both equal. These discussions prove the following.

Theorem 10: Programs 8 and 9 are equivalent.

The equivalence proved in the above theorem demonstrates that the maximum-weight marking problem for nonlive marked graphs possesses the same structure as that for the live marked graphs. 


\section{SUMMARY AND CONCLUSION}

In this paper, we first presented a linear programming formulation of the maximum-weight marking problem on live marked graphs. We have described the details of an algorithm (based on the simplex method) to obtain a maximum-weight marking. The concepts of basic markings and diakoptic firings have been defined. It is shown that each pivot in the simplex method corresponds to a diakoptic firing. An algorithm requiring only vertex firings is given to construct a basic feasible marking from a given initial marking. In addition to constructing a maximumweight marking, our algorithm, as it progresses, constructs a diakoptic firing sequence leading from the initial marking to a maximum-weight marking. We have also introduced the concept of diakoptic firing and established the diakoptic-reachability theorem.

We have given details of an algorithm to construct a maximum-weight marking in the case of capacitated marked graphs. Finally, a formulation of the problem is given in terms of firing counts only. Using this formulation, we have studied the maximum-weight marking problem for the nonlive class of problems. We have shown that the maximum-weight marking problem has the same structure in the cases of both live and nonlive graphs.

We conclude by again pointing out that the problem of determining the maximum resource requirements in the computation graph model of Karp and Miller [9] reduces to the maximum-weight marking problem in the case where the input and the output quanta as well as the threshold of each edge of the computation graph are equal.

\section{REFERENCES}

[1] J. L. Peterson, "Petri nets," Computing Surveys, vol. 9, pp. 223-252, Sept. 1977.

[2] F. Commoner, A. W. Holt, S. Even, and A. Pnueli, "Marked directed graphs," J. Comp. Syst. Sci., vol. 5, pp. 511-523, 1971

[3] T. Murata, "Circuit theoretic analysis and synthesis of marked graphs, IEEE Trans. Circuits Syst., vol. CAS-23, pp. 400-405, July 1977.

[4] M. A. Comeau and K. Thulasiraman, "Algorithms on marked directed graphs," Canadian Electrical Eng. Journal, vol. 9, no. 2, pp. 72-79, April 1984.

[5] S. Kumagai, S. Kodama, and M. Kitagawa, "Submarking reachability of marked graphs," IEEE Trans. Circuits Syst., vol. CAS-31, Feb. 1984.

[6] S. Kumagai, S. Kodama, and M. Kitagawa, "Submarking reachability of marked graphs with token capacity constraints," Trans. IECE Japan, vol. E-67, no. 7, July 1984.

[7] V. Chvatal, Linear Programming. New York: Freeman, 1980.
[8] T. Murata, "Relevance of network theory to models of distributed/parallel processing," J. Franklin Inst., vol. 310, no. 1, pp. 41-50, July 1980 .

[9] R. M. Karp and R. E. Miller, "Properties of a model for parallel computations: determinacy, termination, queueing," SIAM J. Appl. Math., vol. 14, pp. 1390-1411, 1966.

\section{事}

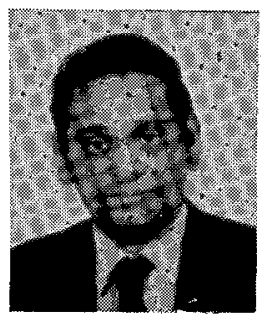

K. Thulasiraman (M'72-SM'84) was born in India in 1942. He received the Bachelor's and Master's degrees in electrical engineering from the University of Madras, Madras, India, in 1963 and 1965 , respectively, and the Ph.D. degree in electrical engineering from the Indian Institute of Technology, Madras, in 1968.

He joined the Indian Institute of Technology, Madras, in 1965, where he was with the Department of Electrical Engineering from 1965 to 1973 and with the Department of Computer Science from 1973 to 1981 . He was promoted to Professor in January 1977. After serving for a year (1981-1982) at the Department of Electrical Engineering, Technical University of Nova Scoita, Halifax, Canada, he joined Concordia University, Montreal, as Professor at the Department of Mechanical Engineering where he was involved in the development of programs in Industrial Engineering at the undergraduate and graduate levels. Since 1984 he has been with the Department of Electrical and Computer Engineering at Concordia University. Earlier, he had held visiting positions at Concordia University during the periods of 1970-1972, 1975-1976, and 1979-1980. He has published over 50 technical papers on different aspects of electrical network theory, graph theory and design and analysis of algorithms. He has also coauthored the book, Graphs, Networks and Algorithms (New York: Wiley-Interscience, 1981). Recently, he has been awarded a Senior Fellowship by the Japan Society for Promotion of Science. Under this fellowship he will be visiting the Tokyo Institute of Technology, Tokyo during March-July, 1988. Dr. Thulasiraman's current research interests are in Network and Systems Theory, Graph Theory, Parallel and Distributed Computations, Operations Research and Computational Graph Theory with Applications in VLSI Design Automation, Computer Networks, Communication Network Planning, etc.

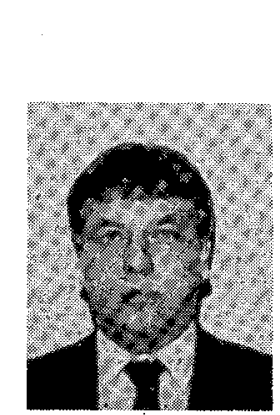

斗

Marc A. Comeau received the Bachelor's degree in electrical engineering from the Technical University of Nova Scotia in 1981 and the Ph.D. degree from Concordia University in 1986.

Dr. Comeau is a researcher at le Centre de Recherche Informatique de Montréal, Inc. (CRIM). He is also associated with Concordia University as an Adjunct Assistant Professor in the Department of Electrical and Computer Engineering. Dr. Comeau's research interests include graph-theoretic modeling, distributed computing, discrete optimization, queueing systems and system performance evaluations. 